\title{
MEMS-based CAOS Smart Camera 177 dB Linear Extreme Dynamic Range Imaging Tests with Calibrated and Controlled Incoherent White Light and Laser Light Targets
}

\author{
Nabeel A. Riza ${ }^{1, *}$, and Mohsin Mazhar ${ }^{1}$ \\ ${ }^{1}$ School of Engineering, University College Cork, Cork, Ireland
}

\begin{abstract}
Highlighted are the linear extreme dynamic range calibrated target imaging tests of the CAOS smart camera showing a $177 \mathrm{~dB}$ linear dynamic range operation with an optical attenuation controlled bright laser target
\end{abstract}

\section{Introduction}

Linear Extreme Dynamic Range (XDR) imaging is required in applications where objects of interest occur in a scene with extreme brightness to weak light zones with low contrast targets of interest embedded within these extreme zones. Recently introduced and demonstrated is the Coded Access Optical Sensor (CAOS) smart camera that has the fundamentals to provide an linear XDR along with a wide spectral response, e.g., $350 \mathrm{~nm}$ to $2700 \mathrm{~nm}$ [1-2]. This smart XDR camera with a linear camera response function is designed on the principles of the XDR RF multi-access wireless mobile network and operates with a variety of image access modes to suit the pixel extraction environment [3]. This camera engages the classic operations CMOS/CCD/FPA sensor to provide a "DC" mode of operation initial image and CAOS timefrequency modulated imaging modes representing the "AC" modes of operations for XDR capture. The CAOS smart camera is an $\mathrm{AC}+\mathrm{DC}$ camera optimized based on imaging conditions and the application. In fact, such optimizations can take place using a variety of computational processing methods, including machine learning. This paper highlights updated processed Dynamic Range (DR) data from the experimental set-up in ref. 4 where the CAOS smart camera underwent linear XDR imaging tests with calibrated and controlled incoherent white light and laser light targets [4].

\section{CAOS Smart Camera Linear Extreme Dynamic Range Imaging Tests}

The details of the CAOS smart camera test conditions are described in ref.4. Highlighted here are the updated processed linear DR imaging results presented as tables and a plot. The LG3 Image Engineering (Germany) lightbox is used as the white light illumination source. A custom 36 patches target with different designed patch
DR values giving an overall $160 \mathrm{~dB}$ test scene $\mathrm{XDR}$ is used to check the designed (D) linear DR values in $\mathrm{dB}$ versus the measured $(\mathrm{M})$ numbers in $\mathrm{dB}$ observed using the CAOS smart camera. Table 1 shows the DR for the CMOS-mode of the camera showing accurate recovery till a DR of $32 \mathrm{~dB}$. Table 2 shows the improved linear imaging DR via the CDMA-mode of the camera showing accurate recovery till a DR of $45.8 \mathrm{~dB}$.

Table 1. CMOS-Mode White Light Imaging DR Data.

\begin{tabular}{|c|c|c|c|c|c|c|c|}
\hline Patch \# & $\mathbf{D}(\mathbf{d B})$ & $\mathbf{M}(\mathbf{d B})$ & $\mathbf{S N R}$ & $\begin{array}{c}\text { Patch } \\
\#\end{array}$ & $\mathbf{D}(\mathbf{d B})$ & $\begin{array}{c}\mathbf{M} \\
(\mathbf{d B})\end{array}$ & SNR \\
\hline 1 & 0 & - & 545 & 11 & 45.8 & 42.28 & 4.2 \\
\hline 2 & 4.6 & 4.69 & 317 & 12 & 50.2 & 45.27 & 3 \\
\hline 3 & 9.2 & 9.19 & 189 & 13 & 54.8 & 48.26 & 2.1 \\
\hline 4 & 13.8 & 12.99 & 122 & 14 & 59.4 & 50.35 & 1.7 \\
\hline 5 & 18.2 & 17.41 & 73 & 15 & 64.0 & 51.72 & 1.4 \\
\hline 6 & 22.8 & 21.94 & 43.5 & 16 & 68.6 & 52.87 & 1.24 \\
\hline 7 & 27.4 & 26.32 & 26.33 & 17 & 73.2 & 54.05 & 1.08 \\
\hline 8 & 32.0 & 30.41 & 16.4 & 18 & 77.8 & 54.63 & 1.012 \\
\hline 9 & 36.6 & 33.72 & 11.2 & 19 & 82.2 & 54.64 & 1.009 \\
\hline 10 & 41.2 & 37.70 & 7.1 & 20 & 86.8 & 54.72 & 1.0001 \\
\hline
\end{tabular}

Table 2. CDMA-Mode White Light Imaging DR Data.

\begin{tabular}{|c|c|c|c|c|c|c|c|}
\hline $\begin{array}{c}\text { Patch } \\
\#\end{array}$ & $\begin{array}{c}\mathbf{D} \\
\text { (dB) }\end{array}$ & $\begin{array}{c}\text { M } \\
\text { (dB) }\end{array}$ & SNR & $\begin{array}{c}\text { Patch } \\
\#\end{array}$ & $\begin{array}{c}\text { D } \\
\text { (dB) }\end{array}$ & $\begin{array}{c}\text { M } \\
\text { (dB) }\end{array}$ & SNR \\
\hline 1 & 0 & - & 1802 & 8 & 32.0 & 32.78 & 41 \\
\hline 2 & 4.6 & 4.21 & 1110 & 9 & 36.6 & 33.65 & 37 \\
\hline 3 & 9.2 & 8.20 & 701 & 10 & 41.2 & 40.27 & 18 \\
\hline 4 & 13.8 & 13.77 & 370 & 11 & 45.8 & 43.8 & 10.6 \\
\hline 5 & 18.2 & 18.28 & 220 & 12 & 50.2 & 44.61 & 5.8 \\
\hline 6 & 22.8 & 22.19 & 140 & 13 & 54.8 & 49.82 & 3.5 \\
\hline 7 & 27.4 & 25.85 & 92 & & & & \\
\hline
\end{tabular}

Table 3 shows the additional improvement in linear DR via the FM-TDMA mode of the camera showing accurate recovery till a DR of $73.2 \mathrm{~dB}$. Experiments indicate that

\footnotetext{
Corresponding author: n.riza@ucc.ie
} 
inter-patch crosstalk at the image plane such as due to camera front lens optics is limiting the higher DR (weaker patch) recovery. Hence the CAOS FM-TDMA mode is reengaged while blocking target plane light from all patches except the patch under measurement.

Table 3. FM-TDMA Mode White Light Imaging DR Data.

\begin{tabular}{|c|c|c|c|c|c|c|c|}
\hline $\begin{array}{c}\text { Patch } \\
\#\end{array}$ & $\begin{array}{c}\mathbf{D} \\
(\mathbf{d B})\end{array}$ & $\begin{array}{c}\mathbf{M} \\
(\mathbf{d B})\end{array}$ & SNR & $\begin{array}{c}\text { Patch } \\
\#\end{array}$ & $\begin{array}{c}\mathbf{D} \\
(\mathbf{d B})\end{array}$ & $\begin{array}{c}\mathbf{M} \\
(\mathbf{d B})\end{array}$ & SNR \\
\hline 1 & 0 & - & 4620 & 10 & 41.2 & 41.10 & 41 \\
\hline 2 & 4.6 & 4.07 & 2891 & 11 & 45.8 & 44.11 & 29 \\
\hline 3 & 9.2 & 9.10 & 1621 & 12 & 50.2 & 49.35 & 16 \\
\hline 4 & 13.8 & 13.83 & 940 & 13 & 54.8 & 53.02 & 10.3 \\
\hline 5 & 18.2 & 18.09 & 576 & 14 & 59.4 & 59.78 & 4.7 \\
\hline 6 & 22.8 & 22.25 & 357 & 15 & 64.0 & 61.05 & 4.1 \\
\hline 7 & 27.4 & 25.23 & 253 & 16 & 68.6 & 67.46 & 2 \\
\hline 8 & 32.0 & 32.71 & 107 & 17 & 73.2 & 70.02 & 1.5 \\
\hline 9 & 36.6 & 34.51 & 87 & 18 & 77.8 & 72.15 & 1.1 \\
\hline
\end{tabular}

Table 4. FM-TDMA Mode XDR Data with patch blocking.

\begin{tabular}{|c|c|c|c|c|c|c|c|}
\hline Patch \# & $\mathbf{D}$ (dB) & $\mathbf{M}$ (dB) & SNR & Patch \# & $\mathbf{D}$ (dB) & $\mathbf{M}$ (dB) & SNR \\
\hline 1 & 0 & - & 100 & 15 & 64.0 & 62 & 97.8 \\
\hline 2 & 4.6 & 4.8 & 98.3 & 16 & 68.6 & 66.4 & 97.5 \\
\hline 3 & 9.2 & 9.3 & 98.9 & 17 & 73.2 & 71.6 & 96 \\
\hline 4 & 13.8 & 13.9 & 97.6 & 18 & 77.8 & 71.1 & 93 \\
\hline 5 & 18.2 & 14.8 & 95.6 & 19 & 82.2 & 80.9 & 88 \\
\hline 6 & 22.8 & 19.2 & 98.3 & 20 & 86.8 & 85.7 & 71 \\
\hline 7 & 27.4 & 23.6 & 98.2 & 21 & 91.4 & 91.1 & 50 \\
\hline 8 & 32.0 & 28.6 & 98.2 & 22 & 96.0 & 95.9 & 34 \\
\hline 9 & 36.6 & 32.3 & 98.1 & 23 & 100.6 & 101.3 & 20 \\
\hline 10 & 41.2 & 39.2 & 98.2 & 24 & 105.2 & 106 & 8.2 \\
\hline 11 & 45.8 & 42.1 & 97.9 & 25 & 109.8 & 111 & 13.3 \\
\hline 12 & 50.2 & 47.2 & 97.7 & 26 & 114.2 & 115.2 & 8.4 \\
\hline 13 & 54.8 & 51.4 & 97.8 & 27 & 118.8 & 124.4 & 7.4 \\
\hline 14 & 59.4 & 57.2 & 98.4 & 28 & 123.4 & 125.3 & 9.4 \\
\hline
\end{tabular}

Table 5. FM-mode XDR Data with a laser patch target.

\begin{tabular}{|c|c|c|c|c|c|c|c|}
\hline $\begin{array}{c}\text { Attenuation } \\
\text { (OD) }\end{array}$ & $\begin{array}{c}\mathbf{D} \\
\text { (dB) }\end{array}$ & $\begin{array}{c}\mathbf{M} \\
\mathbf{( d B )}\end{array}$ & $\mathbf{S N R}$ & $\begin{array}{c}\text { Attenuation } \\
\text { (OD) }\end{array}$ & $\begin{array}{c}\mathbf{D} \\
\mathbf{( d B )}\end{array}$ & $\begin{array}{c}\mathbf{M} \\
\mathbf{( d B )}\end{array}$ & SNR \\
\hline 0 & 0 & - & 269.3 & 6.5 & 130 & 128.1 & 8.4 \\
\hline 1 & 20 & 19.9 & 268.7 & 7.4 & 148 & 147.3 & 5.4 \\
\hline 2 & 40 & 39.3 & 268.7 & 7.7 & 154 & 152.5 & 6.8 \\
\hline 3 & 60 & 57.9 & 268.3 & 7.8 & 156 & 154.7 & 5 \\
\hline 4 & 80 & 80 & 161.3 & 8.4 & 168 & 163.9 & 17.3 \\
\hline 5 & 100 & 97.4 & 36.06 & 8.8 & 176 & 173 & 6.3 \\
\hline 5.5 & 110 & 107.6 & 10.74 & 9 & 180 & 177 & 4 \\
\hline
\end{tabular}

Table 4 shows the further improvement in DR recovery to $123.4 \mathrm{~dB}$. Table 5 shows DR data taken using a laser beam as an Optical Density (OD) controlled single patch up-to a $180 \mathrm{~dB}$ XDR value. These readings are taken using the FM-mode, showing a recovery up-to a $177 \mathrm{~dB}$ XDR. Fig. 1 plot shows the linear $177 \mathrm{~dB}$ XDR response of the demonstrated CAOS smart camera.

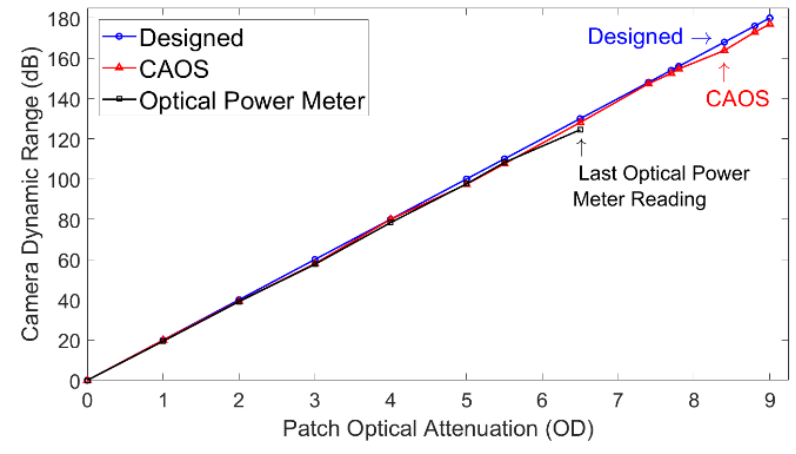

Fig. 1. Linear $177 \mathrm{~dB}$ XDR response using Table 5 Data.

\section{Conclusion}

Calibrated target image testing is a robust method to ascertain the linear DR imaging capability of a camera. Hence highlighted are such initial tests for the CAOS smart camera that both point to the limitations of the testing infrastructure and the deployed camera operations. For example, brightness limits on a pixel basis of the white light LG3 source-based XDR test allowed image recovery to $123.4 \mathrm{~dB}$ DR. The brighter visible laser single patch OD-controlled target allowed linear XDR recovery to $177 \mathrm{~dB}$.

\section{References}

1. N. A. Riza, Coded Access Optical Sensor (CAOS), USA Patent 10,356,392 B2, July 16, 2019.

2. N. A. Riza, "The CAOS Camera Platform - Ushering in a Paradigm Change in Extreme Dynamic Range Imager Design," Invited Paper SPIE OPTO, Vol. 10117, Photonics West, Jan. 30, 2017.

3. N. A. Riza and M. A. Mazhar, "Laser beam imaging via multiple mode operations of the extreme dynamic range CAOS camera," Appl. Opt., Vol. 57, No.E20, 2018

4. N. A. Riza and M. A. Mazhar, "177 dB Linear Dynamic Range Pixels of Interest DSLR CAOS Camera," IEEE Photonics Journal (open access), Volume 11, Number 2, April 2, 2019. 